\title{
Branched Chain Fatty Acids Are Constituents of the Normal Healthy Newborn Gastrointestinal Tract
}

\author{
RINAT R. RAN-RESSLER, SRISATISH DEVAPATLA, PETER LAWRENCE, AND J. THOMAS BRENNA \\ Division of Nutritional Sciences [R.R.R.-R., P.L., J.T.B.], Cornell University, Ithaca, New York 14853; \\ Cayuga Medical Center [S.D.], Ithaca, New York 14850
}

\begin{abstract}
Vernix suspended in amniotic fluid is normally swallowed by the late term fetus. We hypothesized that branched chain fatty acids (BCFA), long known to be major vernix components, would be found in meconium and that the profiles would differ systematically. Vernix and meconium were collected from term newborns and analyzed. BCFA-containing lipids constituted about $12 \%$ of vernix dry weight, and were predominantly saturated, and had 11-26 carbons per BCFA. In contrast, meconium BCFA had 16-26 carbons, and were about $1 \%$ of dry weight. Meconium BCFA were mostly in the iso-configuration, whereas vernix BCFA contained dimethyl and middle chain branching, and five anteiso-BCFA. The mass of BCFA entering the fetal gut as swallowed vernix particles is estimated to be $180 \mathrm{mg}$ in the last month of gestation whereas the total mass of BCFA found in meconium is estimated to be $16 \mathrm{mg}$, thus most BCFA disappear from the fetal gut. The BCFA profiles of vernix and meconium show that BCFA are major components of normal healthy term newborn gastrointestinal tract. BCFA are candidates for agents that play a role in gut colonization and should be considered a nutritional component for the fetus/newborn. (Pediatr Res 64: 605-609, 2008)
\end{abstract}

$\mathrm{B}$ ranched chain fatty acids (BCFA) are mostly saturated fatty acids (SFA) with one or more methyl branches on the carbon chain. BCFA are synthesized mainly by the skin and have long been known to be a major component of vernix caseosa (10-20\% dry weight) (1). Among terrestrial animals, vernix is unique to humans, and is not found in other land mammals, including other primates (2). Vernix is made of sebum and fetal corneocytes $(1,3)$ and is produced by fetal skin starting at $24 \mathrm{wk}$ gestational age and continuing until term birth (4). During the third trimester vernix sloughs off as particulates that become suspended in amniotic fluid $(3,5)$, possibly aided by lung surfactant phospholipids that also enter the amniotic fluid. The fetus normally swallows amniotic fluid in amounts approaching $500 \mathrm{~mL}$ at the end of gestation $(6,7)$ and with it vernix. Thus the late term fetal gut is normally exposed to vernix and its BCFA, increasingly so as parturition approaches.

Vernix dry matter is composed of approximately equal amounts of protein and lipids $(2,8)$. Lipid fractions in vernix have been comprehensively characterized $(9-11)$ and shown to be $25-30 \%$ sterol esters (SE), $18-36 \%$ triglycerides (TAG),

Received May 9, 2008; accepted June 24, 2008.

Correspondence: J. Thomas Brenna, Ph.D., Cornell University, Savage Hall, Ithaca, NY 14853; e-mail: jtb4@ cornell.edu

Supported by NIH grant GM071534
12-16\% wax esters (WE), 9\% squalene, $5 \%$ ceramides. Low levels of nonesterified fatty acid (NEFA) fraction was also detected by some $(10,12)$ but not by others $(13)$. BCFA are found in all acyl-carrying lipid classes, WE (16-53\%) and SE $(27-62 \%)(9-11,13)$, as well as in the TAG $(18-21 \%)$ and NEFA (21\%) fractions (10).

Apart from skin $(1,9,14)$, BCFA are at very low levels in internal tissue (14), but are also found in human milk (15-17) at concentrations as high as $1.5 \% \mathrm{wt} / \mathrm{wt}$ of total fatty acids (FA). This level is comparable with and in some cases greater than that of docosahexaenoic acid (DHA, 22:6n-3) and arachidonic acid (ARA, 20:4n-6) in the same milk. For instance, a 1981 publication reported the concentration of anteiso-17:0 in Australian women's colostrum to be $0.45 \% \mathrm{wt} / \mathrm{wt}$ of total FA, exceeding the concentrations of DHA $(0.32 \% \mathrm{wt} / \mathrm{wt})$ and ARA (0.4\%wt/wt) (17).

Meconium, the newborn's first fecal pass, first appears in the fetal GI tract at around 12 wk of gestational age, and is normally passed after birth (18-20). It consists of amniotic fluid residue, skin and gastrointestinal (GI) epithelial cells, GI secretions and enzymes, lipids, sugars, proteins, cholesterol, sterols, bile acid and salts $(18,19,21,22)$. Meconium contains $12 \%$ dry weight lipid (21), and there is only one unconfirmed study reporting BCFA in meconium (23). There are no studies linking BCFA composition of vernix and meconium in the same infants.

We hypothesized that vernix BCFA of term newborns would survive the alimentary canal and be found in meconium. Our test of this hypothesis led us to characterize the relative BCFA profiles of vernix and meconium to establish the degree to which the profile is altered by the sterile fetal gut in utero.

\section{METHODS}

This study was approved by the Cornell University and the Cayuga Medical Center Institutional Review Boards (IRB) on the Use of Human Subjects. The IRB approved an exemption from the requirement to obtain individual informed consent because the human materials sampled, vernix and meconium, are deemed to be medical waste and no individually identifiable information was obtained from participants.

Sample collection. Eighteen samples of vernix and meconium were collected from 18 normal term newborns at Cayuga Medical Center in Ithaca, NY. Vernix was removed from the shoulder regions in the birthing room,

Abbreviations: BCFA, branched-chain fatty acids; GC, gas chromatography; GI, gastrointestinal; FA, fatty acids; FAME, fatty acid methyl esters; MS, mass spectrometry; MUFA, monounsaturated fatty acids; SE, sterol esters; SFA, saturated fatty acids; TAG, triacylglycerol; WE, wax esters 
placed in clean tubes and stored at $-80^{\circ} \mathrm{C}$ until analysis. Meconium was collected from diapers and similarly transferred into clean tubes and stored at $-80^{\circ} \mathrm{C}$ until analysis.

FA analysis. Total lipids were extracted from the vernix and the meconium samples according to a modified Bligh and Dyer method (24). Fatty acids are overwhelmingly found in mammalian pools, such as vernix and meconium, as acyl moieties, which are constituents of higher molecular weight lipid molecules such as TAG, SE, and WE. For detailed molecular analysis, fatty acyl groups are hydrolyzed and fatty acid methyl esters (FAME) synthesized for analysis. FAME were prepared using 14\% BF3 in methanol (Sigma Chemical Co. Chemical, St. Louis, MO). Butylated hydroxytoluene (BHT) was added to methanol as an antioxidant. Heptadecanoic acid (Sigma Chemical Co. Chemical, St. Louis, MO) in chloroform was used as an internal standard. This routine step obscures heptadecanoic acid, which is normally rare in mammalian tissue but is present in vernix and meconium. Because of the extraordinary diversity of FA in these samples, any internal standard interferes with analysis of one or more FA in some of the samples. A correction was applied to estimate the extent of interference, and the signals were carefully calibrated against external standards.

FAME analyses were performed using a Hewlett Packard 5890 series II gas chromatograph (GC). A BPX-70 column $(60 \mathrm{~m} \times 0.32 \mathrm{~mm} \times 0.25 \mu \mathrm{m}$, SGE, Austin, TX) was used for the analysis with $\mathrm{H}_{2}$ as the carrier gas. FAME identities were determined by a chemical ionization (CI) and electron impact (EI) mass spectrometry (MS), using a Varian Star 3400 GC coupled to a Varian Saturn 2000 ion trap MS. BCFA FAME identities were based on GC retention time of each substance and its electron impact mass spectra. FAME mass spectral assignments were confirmed by conversion of the FAME to picolinyl ester derivatives according to the method described by Christie (available online at www.lipidlibrary.co.uk), and Yang and coworkers (25), followed by GC/MS analysis and comparison to literature spectra (26-31).

An equal weight FAME mixture (68A; Nuchek Prep, Elysian, MN) was used to calculate response factors. The following were also used as standards: n-11:0 up to n-24:0 (Nuchek Prep, Elysian, MN); iso-13:0, anteiso-13:0, iso15:0, anteiso-15:0; iso-17:0, anteiso-17:0 (Larodan Fine Chemicals AB, Malmo, Sweden) and 10 methyl hexadecanoic acid (Matreya LLC, Pleasant Gap, PA). FA levels were expressed as weight $\%$ of total fatty acids for all 11-32 carbons FA.

Statistics. Data are expressed as mean \pm SD for study population characteristics, and as mean \pm SEM for FA analysis. Statistical analyses were made using JMP 6 (SAS Institute, Cary, NC). Differences in mean of each FA were calculated using one sample $t$ test for nonzero differences, with $p<0.05$ declared significant.

\section{RESULTS}

Subjects. Characteristics of the study population are presented in Table 1. No complications were present for any of the newborns other than as noted. All but two newborns were by vaginal delivery. Six mothers received antibiotic treatment during pregnancy; five of them gave birth to female infants.

Overall FA distribution. A profile of FA classes is shown in Table 2. Comparisons of all classes were significant at the

\begin{tabular}{ccc} 
Table 1. Characteristics of study & population [mean $\pm S D($ range)] \\
\hline Mother's age (y) & $29 \pm 5.8$ & $18-42$ \\
Gestational age (wk) & $40 \pm 1$ & $38-41$ \\
Birthweight $(\mathrm{kg})$ & $3.3 \pm 0.5$ & $2.3-4.4$ \\
Gender & 10 female, 8 males \\
Delivery & 16 vaginal, 2 CS* \\
Antibiotics & 5 female, 1 male
\end{tabular}

\footnotetext{
* Cesarean section.
}

Table 2. Profile of FA classes (\%w/w) in vernix and meconium (mean \pm SEM)

\begin{tabular}{lrr}
\hline \multicolumn{1}{c}{ FA } & \multicolumn{1}{c}{ Vernix* } & Meconium \\
\hline BCFA & $29.1 \pm 1.5$ & $17.5 \pm 1.3$ \\
n-SFA & $34.0 \pm 1.9$ & $51.3 \pm 3.0$ \\
n-MUFA & $31.0 \pm 1.7$ & $22.4 \pm 2.1$ \\
PUFA & $3.9 \pm 0.4$ & $7.1 \pm 1.1$ \\
\hline
\end{tabular}

\footnotetext{
$* p<0.05$
}

$p<0.05$ level. BCFA constituted almost a third $(29.1 \pm$ $1.5 \% \mathrm{wt} / \mathrm{wt}$ ) of all FA in vernix and were significantly higher compared with the mean levels in meconium $(17.5 \pm 1.3 \% \mathrm{wt} /$ $\mathrm{wt} ; p<0.05)$. This drop in BCFA was accompanied by a reciprocal increase in normal (n-) saturated FA (n-SFA) specifically, $34 \pm 1.9 \% \mathrm{wt} / \mathrm{wt}$ in vernix and $51.3 \pm 3.0 \% \mathrm{wt} / \mathrm{wt}$ in meconium $(p<0.05)$. Differences in $n$-monounsaturated fatty acids (MUFA) and polyunsaturated fatty acid (PUFA) were modest by comparison. Overall, BCFA hydrolyzed from their native lipid classes constituted $5.8 \%$ of dry weight of vernix, corresponding to approximately $12 \%$ of dry weight of vernix within the native BCFA-containing lipids. Meconium had $0.55 \%$ dry weight of hydrolyzed BCFA and an estimated $1 \%$ of BCFA-containing lipids.

BCFA distribution in vernix and meconium. Fig. 1 is a graphical summary of the BCFA profiles for vernix and meconium for those BCFA detected in samples from at least three newborns, presented left to right in order of carbon number. In total, 30 BCFA were identified in vernix whereas nine were also detected in meconium. Vernix BCFA ranged from 11 to 26 carbon atoms and were primarily saturated apart from two iso-monounsaturates. Iso-BCFA, anteiso-BCFA, middle chain monomethyl BCFA and dimethyl BCFA were all detected among vernix BCFA. In contrast, meconium BCFA had a much more restricted range of carbon numbers, from 16 to 26 carbons. Of the nine meconium BCFA, eight were iso-BCFA, of which two were MUFA, and one was anteiso.

The profile of the iso-BCFA in vernix and meconium is shown in Fig. 2. The vernix iso-BCFA profile had odd and even carbon numbered FA from iso-12:0 to iso-16:0, and only even carbon numbers at greater chain lengths. In contrast, meconium iso-BCFA was dominated by the shortest chain BCFA in its profile, iso-16:0, which was more than twice the relative concentration of any other BCFA. Five of the eight iso-BCFA appearing in both vernix and meconium were a significantly different proportion of BCFA in the respective profiles; the preponderance of longer chains in meconium lead to significant differences in three of the four iso-BCFA of chain numbers from 20 to 26 carbons.

The profile of anteiso-BCFA is shown in Fig. 3. All five anteiso-BCFA detected in vernix are odd carbon numbered. They ranged from 13 to 25 carbons, and only one, anteiso17:0, was found in meconium.

Fig. 4 is a graphical summary of the straight chain, $n$-FA, profiles for vernix and meconium. Vernix normal FA had 11-26 carbon atoms, and meconium FA had 14-26 carbons, and both contained small amounts of odd chain number FA. Again, meconium BCFA tended to be of greater molecular weight.

\section{DISCUSSION}

The presence of BCFA in both vernix and meconium of healthy term infants indicates that BCFA are a major component of gut contents of normal term newborns, and their presence in meconium implies that they are present throughout the length of the gut. As such, BCFA are a component of the GI tract milieu present when the first few environmental 


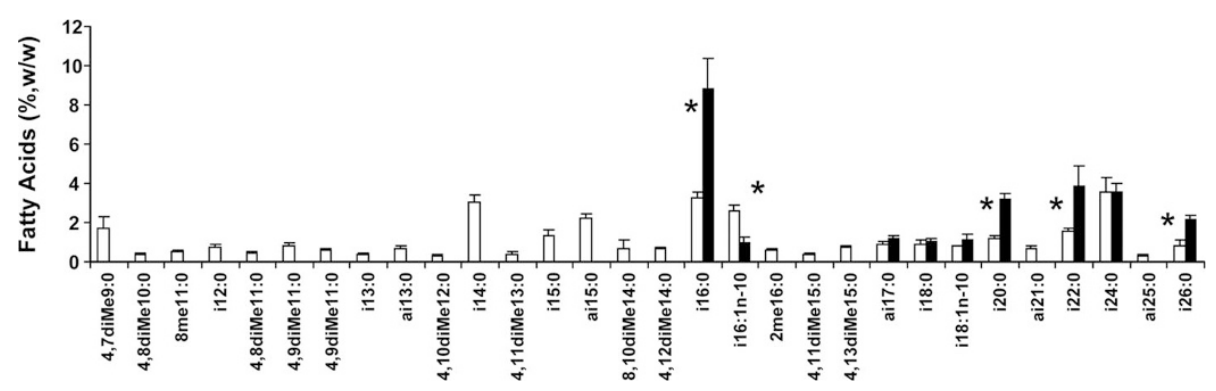

Figure 1. BCFA methyl esters profiles for vernix and meconium (means \pm SEM, $n \leq 18$ newborn) listed left to right in order of molecular weight. Means are for those FA appearing in samples from at least three newborns. iso-BCFA have a dimethyl terminal structure: iso-16:0 is synonymous with 14-methyl-15:0 (14-methyl-pentadecanoic acid). anteiso-BCFA have a methyl branch at the n-2 position: anteiso-17:0 is synonymous with 14-methyl-16:0 (14-methylhexadecanoic acid). $\mathrm{i}=$ iso; ai $=$ anteiso; $\mathrm{Me}=$ methyl; diMe $=$ two methyl branches. Key: $\square$ vernix; $\square$ meconium. $* p<0.05$.

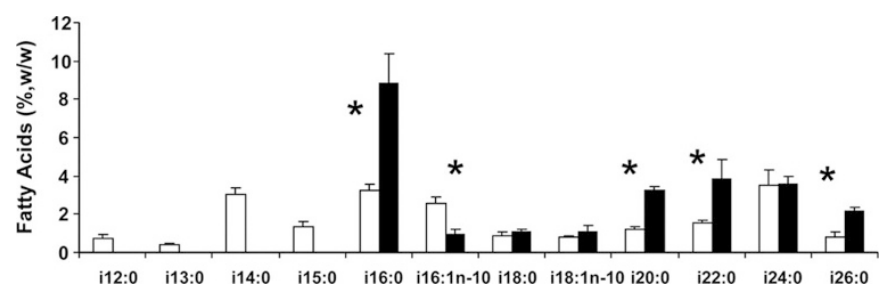

Figure 2. Iso-BCFA methyl esters in vernix and meconium (means \pm SEM, $n \leq 18$ newborn) listed left to right in order of molecular weight. $\mathrm{i}=$ iso. Key: $\square$ vernix; $\square$ meconium. ${ }^{*} p<0.05$.

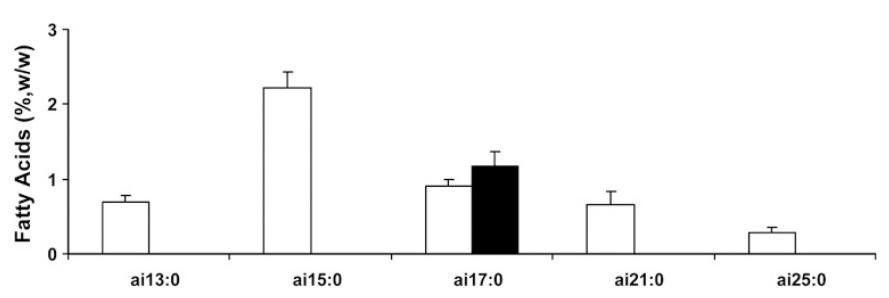

Figure 3. Anteiso-BCFA methyl esters in vernix and meconium (means \pm SEM, $n \leq 18$ newborn) listed left to right in order of molecular weight. ai $=$ anteiso. Key: $\square$ vernix; $\square$ meconium.

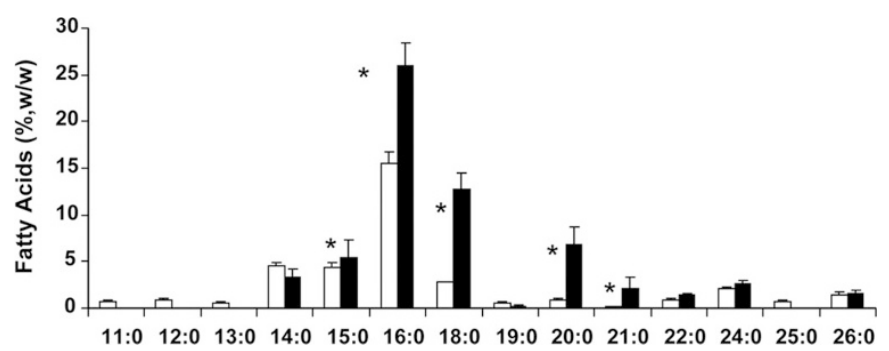

Figure 4. Normal (straight chain) FAME in vernix and meconium (means \pm SEM, $n \leq 18$ newborn) listed left to right in order of molecular weight. Key: $\square$ vernix; $\square$ meconium. $* p<0.05$.

microorganisms appear in the initial stage of gut colonization during and immediately after parturition or cesarean section. In meconium, the systematic shift in BCFA profiles to high molecular weights, as well as the absence of most BCFA other than iso-BCFA, indicates that the fetal alimentary canal readily absorbs and metabolizes most BCFA.

There are many reports of BCFA in breastmilk, with the earliest and most extensive, showing 54 BCFA with a cumulative concentration of $1.5 \% \mathrm{wt} / \mathrm{wt}$ (16). A 1981 paper measured the concentration of BCFA in mature Australian breast milk to be a total of $0.84 \% \mathrm{wt} / \mathrm{wt}$ and one BCFA, anteiso-17:0 in colostrum at $0.45 \% \mathrm{wt} / \mathrm{wt}$ of fatty acids, exceeding the concentrations of DHA $(0.32 \% \mathrm{wt} / \mathrm{wt})$ and ARA $(0.40 \% \mathrm{wt} / \mathrm{wt})$ in the same mother's mature breastmilk (17). Chen reported four BCFA with a cumulative concentration of $0.58 \%$, wt $/ \mathrm{wt}$ in Canadian breastmilk (32). A study of California women yielded an average of $0.60 \%$ BCFA for the four BCFA that were reported $(15: 0,16: 0,17: 0,18: 0$, branch location not reported) (33). Corso found six BCFA in milks of 40 women in southern Italy (34), with anteiso-17:0 ranging from 0.12 to $0.93 \%$ of total fatty acids. This variability is not unlike that for DHA; breastmilk DHA ranges between $0.06 \% \mathrm{wt} / \mathrm{wt}$ and $1.40 \% \mathrm{wt} / \mathrm{wt}$ (35). Many, but not all, breastmilk fatty acid concentrations are closely linked to the dietary intake of the fatty acid or its precursor, including DHA to which the wide reported range is ascribed (35). It is most likely that the absence of BCFA identification in most breast milk FA papers is due to their low concentration, their appearance in a GC trace amid the major saturates and monounsaturates in the chromatogram, and the historical absence of a compelling metabolic role for them.

The exposure of the gut to BCFA in utero, and possibly from breastmilk, is greater than any other period of life because BCFA are at trace levels in normal foods. The unique niche represented by BCFA and other components in the human gut may be important for establishing commensal bacteria during colonization. BCFA are prominent membrane components of many bacterial species $(36,37)$. For instance, BCFA constitute $95 \%$ of the FA in several bacilli and lactobacilli, including Sporolactobacillus inulinus, which has very recently been shown to be a probiotic candidate (37). The FA of nine Bifidobacterium strains include BCFA such as iso14:0, anteiso-15:0, iso-16:0 and iso-18:0 at various levels $(0.6-24.6 \% \mathrm{wt} / \mathrm{wt})$. Iso-14:0 is the second most abundant FA in Bifidobacterium breve with levels as high as $24.6 \% \mathrm{wt} / \mathrm{wt}$ (38). It is reasonable to hypothesize that the presence of BCFA in the neonatal gut would alter the mix of dominant species, favoring those organisms that use BCFA in their membranes, and we postulate that BCFA are a unique feature of the human fetal gut favoring the growth of commensal bacteria during colonization.

This hypothesis has implications for colonization of the GI tract of very premature infants, and may be a factor in the development of necrotizing enterocolitis (NEC), the etiology and pathogenesis of which is not well understood $(39,40)$. 
NEC is one of the major causes of morbidity in premature infants (39) and it is certainly related to pathogen overgrowth (41). Leading hypotheses with empirical support are that NEC is related to prematurity, enteral feeding, and bacterial colonization (40). Importantly, it has not been observed prenatally. NEC risk is higher among lower gestational age infants and is rare in term infants (42). Breastmilk consumption is associated with a lower incidence of NEC $(40,43)$. Although no specific pathogenic bacteria has been associated with NEC (43), supplementation of premature animals and infants with probiotic strains appear to reduce its incidence $(39,44)$. With these considerations, we hypothesize that BCFA have a role in enhancing proper GI colonization: vernix begins to appear around wk 24 of gestation and accumulates as particulates in amniotic fluid toward term (3) thus the GI tract of very premature infants is not exposed to vernix BCFA prenatally. Postnatally they would be exposed to BCFA if breastfed, but formula-fed preterms would not be exposed to BCFA since they are not a component of preterm formulas. Finally, we note that the incidence of NEC drops as gestational age approaches normal term, therefore later term premature infants would be exposed to some BCFA and may benefit if the hypothesis is correct.

We can estimate the mass of BCFA entering and exiting the alimentary canal. At term, amniotic fluid lipids are about 154 $\mathrm{mg} / \mathrm{L}$ (45), of which about $52 \mathrm{mg} / \mathrm{L}$ are phospholipids that are likely to originate as BCFA-free lung surfactant $(3,10)$. Thus the amniotic fluid vernix FA concentration is about $102 \mathrm{mg} / \mathrm{L}$. Of this, our measurements indicate that $57 \%$ are FA, to yield $58 \mathrm{mg} / \mathrm{L}$. Our data (Table 2) further indicate that $29 \%$ are BCFA, to yield $17 \mathrm{mg} / \mathrm{L}$ BCFA. The fetus is estimated to swallow $200-500 \mathrm{~mL} / \mathrm{d}$ of amniotic fluid near term $(46,47)$, and taking the midpoint of this range, $350 \mathrm{~mL} / \mathrm{d}, 6 \mathrm{mg} \mathrm{BCFA}$ per day enter the fetal GI tract amounting to $30 \times 6=180 \mathrm{mg}$ BCFA in the last month of gestation. Meconium is the output of the GI tract integrated from about 12 wk gestation. Total meconium for 27 term infants was reported (48) to be $8.9 \mathrm{~g}$ wet weight, averaging $32 \%$ dry weight, or $2.8 \mathrm{~g}$. Our data indicate that about $0.55 \%$ is BCFA, or about $16 \mathrm{mg}$ average total BCFA in meconium. This value is an order of magnitude lower than our estimate of the BCFA swallowed in the last month of gestation, and suggests that most of the BCFA disappear during transit. The distribution and structural characteristics of BCFA that do appear in meconium reflect processing of vernix by the enterocytes. Our data show that C12-15 BCFA, as well as nearly all BCFA apart from isoBCFA, are absent from meconium and thus must have been metabolized. The nature of this metabolism remains to be determined, in part because BCFA and their interaction with human enterocytes have not been studied.

Chain elongation is one likely metabolic transformation that would explain the absence of C12-15 BCFA, and preponderance of longer chain BCFA, in meconium. Suggestive evidence in support of this hypothesis is found in the data of Fig. 1. The significantly greater level of meconium iso-16:0 compared with vernix iso-16:0, is roughly the sum of vernix iso-14:0 and iso-16:0, consistent with the hypothesis that elongation of vernix iso-14:0 adds to the existing iso-16:0.
Similar observations apply to meconium iso-20:0 and vernix iso-18:0 and iso-20:0.

Medium chain fatty acids (C8-C14) are commonly fed to premature infants because they are efficiently absorbed through the gastric mucosa, directly transported to the liver via the portal vein, and oxidized by the immature GI tract. Although the BCFA with 15 or fewer carbons are absent from meconium, Fig. 4 shows that the FA $n-14: 0$ and $n-15: 0$ are partially excreted. This observation implies that there is selective uptake and retention of BCFA by the fetal GI tract that may not operate as efficiently for the $n$-FA.

Our measurements of BCFA were in line with previous data. BCFA constituted almost one third of all FA in vernix $(11,49)$, and the levels of vernix SFA, MUFA and PUFA were within the range encompassed by previous reports $(10,49,50)$. We found only odd numbered carbon anteiso-BCFA, consistent with some previous reports (49-51), but not with others $(10,11)$. BCFA averaged $17 \% \mathrm{wt} / \mathrm{wt}$ of all FA in meconium in our samples. The single previous study showing BCFA in meconium reported only on the FFA fraction and used GC with retention time matching for identification. Iso-FA with 22 and 24 carbons were identified at $4 \% \mathrm{wt} / \mathrm{wt}$ and $6 \% \mathrm{wt} / \mathrm{wt}$, respectively, and nine other iso-BCFA were tentatively assigned $(\mathrm{C} 14-21,25)$ with no percent fraction provided.

Although five anteiso-BCFA were detected in vernix, anteiso-17:0 was the sole anteiso-BCFA detected in meconium, and there is no obvious explanation as to why this was the case. Weanling rats fed $100 \mathrm{mg} / \mathrm{wk}$ anteiso-17:0 in an otherwise fat free diet excreted $8-10 \%$ in the feces and stored a similar amount in adipose tissue (52), and apparently also converted a small amount anteiso-15:0. The remaining 80\% was metabolized to substances other than anteiso-FA. The levels of anteiso-17:0 have been reported to be the highest among all BCFA in at least one study of breastmilk (17), and it is notable that anteiso-17:0 is a major lipids constituent of many bacterial membrane (36).

The combined levels of the middle chain monomethyl and dimethyl BCFA in our vernix samples were similar to the levels reported in a single vernix sample by Nicolaides and Apon (49). In our sample of 18 newborns, the average proportions of dimethyl BCFA dominated over middle chain monomethyl BCFA. The first methyl branch in the dimethyl BCFA was located predominantly on the fourth carbon of the chain, consistent with previous findings $(49,50)$. However, in our study, the second methyl branch in half of the dimethyl BCFA was located on an odd numbered carbon, and in almost all the dimethyl BCFA, this methyl branch was located on the anteiso-carbon of the FA chain.

In summary, there are dramatic and systematic differences in BCFA composition between vernix and meconium, indicating that BCFA are actively metabolized in the fetal GI tract. This observation implies that vernix should be considered a nutritional agent, and that BCFA are a normal and quantitatively substantial component of the normal term newborn GI tract. Further studies are warranted to understand the uptake and metabolism of BCFA by enterocytes, and the role of BCFA during bacterial colonization. The absence of vernix, and BCFA, in the GI tract of very premature, formula-fed 
infants may have a role in the etiology of NEC, among the most devastating conditions facing the preterm infant.

\section{REFERENCES}

1. Nicolaides N, Ray T 1965 Skin Lipids. 3. Fatty chains in skin lipids. The use of vernix caseosa to differentiate between endogenous and exogenous components in human skin surface lipid. J Am Oil Chem Soc 42:702-707

2. Pickens WL, Warner RR, Boissy YL, Boissy RE, Hoath SB 2000 Characterization of vernix caseosa: water content, morphology, and elemental analysis. J Invest Dermatol 115:875-881

3. Narendran V, Wickett RR, Pickens WL, Hoath SB 2000 Interaction between pulmonary surfactant and vernix: a potential mechanism for induction of amniotic fluid turbidity. Pediatr Res 48:120-124

4. Moore C, Dempsey D, Deitermann D, Lewis D, Leikin J 1996 Fetal cocaine exposure: analysis of vernix caseosa. J Anal Toxicol 20:509-511

5. Yoshio H, Tollin M, Gudmundsson GH, Lagercrantz H, Jornvall H, Marchini G, Agerberth B 2003 Antimicrobial polypeptides of human vernix caseosa and amniotic fluid: implications for newborn innate defense. Pediatr Res 53:211-216

6. Miettinen TA, Luukkainen T 1968 Gas-liquid chromatographic and mass spectrometric studies on sterols in vernix caseosa, amniotic fluid and meconium. Acta Chem Scand 22:2603-2612

7. Sherman DJ, Ross MG, Day L, Ervin MG 1990 Fetal swallowing: correlation of electromyography and esophageal fluid flow. Am J Physiol 258:R1386-R1394

8. Hoeger PH, Schreiner V, Klaassen IA, Enzmann CC, Friedrichs K, Bleck O 2002 Epidermal barrier lipids in human vernix caseosa: corresponding ceramide pattern in vernix and fetal skin. Br J Dermatol 146:194-201

9. Nicolaides N, Fu HC, Ansari MN, Rice GR 1972 The fatty acids of wax esters and sterol esters from vernix caseosa and from human skin surface lipid. Lipids 7:506-517

10. Rissmann R, Groenink HW, Weerheim AM, Hoath SB, Ponec M, Bouwstra JA 2006 New insights into ultrastructure, lipid composition and organization of vernix caseosa. J Invest Dermatol 126:1823-1833

11. Kaerkkaeinen J, Nikkari T, Ruponen S, Haahti E 1965 Lipids of Vernix Caseosa. J Invest Dermatol 44:333-338

12. Tollin M, Bergsson G, Kai-Larsen Y, Lengqvist J, Sjovall J, Griffiths W, Skuladottir GV, Haraldsson A, Jornvall H, Gudmundsson GH, Agerberth B 2005 Vernix caseosa as a multi-component defence system based on polypeptides, lipids and their interactions. Cell Mol Life Sci 62:2390-2399

13. Nazzaro-Porro M, Passi S, Boniforti L, Belsito F 1979 Effects of aging on fatty acids in skin surface lipids. J Invest Dermatol 73:112-117

14. Nicolaides N 1974 Skin lipids: their biochemical uniqueness. Science 186:19-26

15. Jensen RG 1995 Handbook of Milk Composition. San Diego: Academic Press Inc

16. Egge H, Murawski U, Ryhage R, Gyorgy P, Chatranon W, Zilliken F 1972 Minor constituents of human milk. IV. Analysis of the branched chain fatty acids. Chem Phys Lipids 8:42-55

17. Gibson RA, Kneebone GM 1981 Fatty acid composition of human colostrum and mature breast milk. Am J Clin Nutr 34:252-257

18. Ahanya SN, Lakshmanan J, Morgan BL, Ross MG 2005 Meconium passage in utero: mechanisms, consequences, and management. Obstet Gynecol Surv 60:45-56; quiz $73-44$

19. Gareri J, Klein J, Koren G 2006 Drugs of abuse testing in meconium. Clin Chim Acta 366:101-111

20. Ostrea EM Jr, Hernandez JD, Bielawski DM, Kan JM, Leonardo GM, Abela MB, Church MW, Hannigan JH, Janisse JJ, Ager JW, Sokol RJ 2006 Fatty acid ethyl esters in meconium: are they biomarkers of fetal alcohol exposure and effect? Alcohol Clin Exp Res 30:1152-1159

21. Buchanan DJ, Rapoport S 1952 Chemical comparison of normal meconium and meconium from a patient with meconium ileus. Pediatrics 9:304-310

22. Righetti C, Peroni DG, Pietrobelli A, Zancanaro C 2003 Proton nuclear magnetic resonance analysis of meconium composition in newborns. J Pediatr Gastroenterol Nutr 36:498-501

23. Terasaka D, Clark DA, Singh BN, Rokahr J 1986 Free fatty acids of human meconium. Biol Neonate 50:16-20

24. Bligh EG, Dyer WJ 1959 A rapid method of total lipid extraction and purification Can J Biochem Physiol 37:911-917

25. Serhan CN, Gotlinger K, Hong S, Lu Y, Siegelman J, Baer T, Yang R, Colgan SP, Petasis NA 2006 Anti-inflammatory actions of neuroprotectin D1/protectin D1 and its natural stereoisomers: assignments of dihydroxy-containing docosatrienes. J Immunol 176:1848-1859

26. Carballeira NM, Miranda C, Lozano CM, Nechev JT, Ivanova A, Ilieva M, Tzvetkova I, Stefanov K 2001 Characterization of novel methyl-branched chain fatty acids from a halophilic Bacillus species. J Nat Prod 64:256-259

27. Suutari M, Laakso S 1993 Signature GLC-MS ions in identification of [Delta]5- and [Delta]9 unsaturated iso- and anteiso-branched fatty acids. J Microbiol Methods 17:39-48

28. Karlsson H, Odham G 1969 Studies on feather waxes of birds. VIII. Composition of the wax in the free-flowing secretion from the preen gland of the oystercatcher (Haematopus ostralegus) Ark Kemi 31:143-158

29. Yu QT, Liu BN, Zhang JY, Huang ZH 1988 Location of methyl branchings in fatty acids: fatty acids in uropygial secretion of Shanghai duck by GC-MS of 4,4dimethyloxazoline derivatives. Lipids 23:804-810

30. Apon JM, Nicolaides N 1975 The determination of the position isomers of the methyl branches fatty acids methyl esters by capillary GC/MS. J Chromatogr Sci 13:467-473

31. Harvey DJ 1989 Identification of long-chain fatty acids and alcohols from human cerumen by the use of picolinyl and nicotinate esters. Biomed Environ Mass Spectrom 18:719-723

32. Chen ZY, Pelletier G, Hollywood R, Ratnayake WM 1995 Trans fatty acid isomers in Canadian human milk. Lipids 30:15-21

33. Aitchison JM, Dunkley WL, Canolty NL, Smith LM 1977 Influence of diet on trans fatty acids in human milk. Am J Clin Nutr 30:2006-2015

34. Corso G, Colavita C, Esposito M, Roma R, Napoli C, Zamparelli M, Ansanelli V 1995 Gaschromatography-mass spectrometry analysis of fatty acids in human milk from forty puerperae living in southern Italy. Riv Eur Sci Med Farmacol 17:215-219

35. Brenna JT, Varamini B, Jensen RG, Diersen-Schade DA, Boettcher JA, Arterburn LM 2007 Docosahexaenoic and arachidonic acid concentrations in human breas milk worldwide. Am J Clin Nutr 85:1457-1464

36. Kaneda T 1991 Iso- and anteiso-fatty acids in bacteria: biosynthesis, function, and taxonomic significance. Microbiol Rev 55:288-302

37. Huang HY, Huang SY, Chen PY, King VA, Lin YP, Tsen JH 2007 Basic characteristics of Sporolactobacillus inulinus BCRC 14647 for potential probiotic properties. Curr Microbiol 54:396-404

38. Veerkamp JH 1971 Fatty acid composition of Bifidobacterium and Lactobacillus strains. J Bacteriol 108:861-867

39. Caplan MS, Miller-Catchpole R, Kaup S, Russell T, Lickerman M, Amer M, Xiao Y, Thomson R Jr 1999 Bifidobacterial supplementation reduces the incidence of necrotizing enterocolitis in a neonatal rat model. Gastroenterology 117:577-583

40. Claud EC, Walker WA 2001 Hypothesis: inappropriate colonization of the premature intestine can cause neonatal necrotizing enterocolitis. FASEB J 15:1398-1403

41. Hallstrom M, Eerola E, Vuento R, Janas M, Tammela O 2004 Effects of mode of delivery and necrotising enterocolitis on the intestinal microflora in preterm infants. Eur J Clin Microbiol Infect Dis 23:463-470

42. Beeby PJ, Jeffery H 1992 Risk factors for necrotising enterocolitis: the influence of gestational age. Arch Dis Child 67:432-435

43. Lucas A, Cole TJ 1990 Breast milk and neonatal necrotising enterocolitis. Lancet 336:1519-1523

44. Hoyos AB 1999 Reduced incidence of necrotizing enterocolitis associated with enteral administration of Lactobacillus acidophilus and Bifidobacterium infantis to neonates in an intensive care unit. Int J Infect Dis 3:197-202

45. Biezenski JJ, Pomerance W, Goodman J 1968 Studies on the origin of amniotic fluid lipids. I. Normal composition. Am J Obstet Gynecol 102:853-861

46. Pritchard JA 1965 Deglutition by normal and anencephalic fetuses. Obstet Gynecol 25:289-297

47. Pritchard JA 1966 Fetal swallowing and amniotic fluid volume. Obstet Gynecol 28:606-610

48. Friel JK, Matthew JD, Andrews WL, Skinner CT 1989 Trace elements in meconium from preterm and full-term infants. Biol Neonate 55:214-217

49. Nicolaides N, Apon JM 1976 Further studies of the saturated methyl branched fatty acids of vernix caseosa lipid. Lipids 11:781-790

50. Nicolaides N 1971 The structures of the branched fatty acids in the wax esters of vernix caseosa. Lipids 6:901-905

51. Haahti E, Nikkari T, Salmi AM, Laaksonen AL 1961 Fatty acids of vernix caseosa Scand J Clin Lab Invest 13:70-73

52. Livingston M, Bell ME, Shorland FB, Gerson T, Hansen RP 1957 The metabolism in the rat of naturally occurring $(+)-14$-methylhexadecanoic acid. Biochem $\mathrm{J}$ $65: 438-440$ 